\title{
Bleeding after Endoscopic Resection in Patients with End-Stage Renal Disease on Dialysis: A Multicenter Propensity Score-Matched Analysis
}

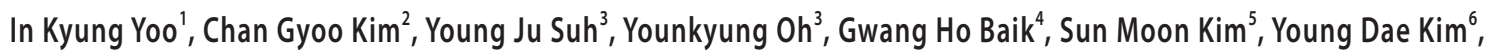 \\ Chul-Hyun Lim ${ }^{7}$, Jung Won Jeon ${ }^{8}$, Su Jin Hong ${ }^{9}$, Byoung Wook Bang ${ }^{10}$, Joon Sung Kim ${ }^{11}$ and Jun-Won Chung ${ }^{12}$ \\ ${ }^{1}$ Department of Gastroenterology, Cha Bundang Medical Center, Cha University College of Medicine, Seougnam, ${ }^{2}$ Center for Gastric Cancer, \\ National Cancer Center, Goyang, ${ }^{3}$ Department of Biomedical Sciences, College of Medicine, Inha University, Incheon, ${ }^{4}$ Department of \\ Internal Medicine, Hallym University College of Medicine, Chuncheon Sacred Heart Hospital, Chuncheon, ${ }^{5}$ Department of Internal Medicine, \\ Konyang University Hospital, Seoul, ${ }^{6}$ Department of Internal Medicine, Chosun University School of Medicine, Gwangju, ${ }^{7}$ Department \\ of Internal Medicine, The Catholic University of Korea, Seoul St. Mary's Hospital, Seoul, ${ }^{8}$ Department of Internal Medicine, Kyung Hee \\ University Hospital at Gandong, College of Medicine, Seoul, ${ }^{9}$ Department of Internal Medicine, Soonchunhyang University School of \\ Medicine, Bucheon, Seoul, ${ }^{10}$ Department of Internal Medicine, Inha University College of Medicine, Incheon, ${ }^{11}$ Department of Internal \\ Medicine, Incheon St. Mary's Hospital, College of Medicine, The Catholic University of Korea, Seoul, ${ }^{12}$ Department of Internal Medicine, \\ Gachon University, Gil Medical Center, Incheon, Korea
}

Background/Aims: Frequent bleeding after endoscopic resection (ER) has been reported in patients with end-stage renal disease (ESRD). We aimed to evaluate the association and clinical significance of bleeding with ER in ESRD patients on dialysis.

Methods: Between February 2008 and December 2018, 7,571 patients, including 47 ESRD patients on dialysis who underwent ER for gastric neoplasia, were enrolled. A total of 47 ESRD patients on dialysis were propensity score-matched 1:10 to 470 non-ESRD patients, to adjust for between-group differences in variables such as age, sex, comorbidities, anticoagulation use, tumor characteristics, and ER method. Matching was performed using an optimal matching algorithm. For the matched data, clustered comparisons were performed using the generalized estimating equation method. Medical records were retrospectively reviewed. Frequency and outcomes of post-ER bleeding were evaluated.

Results: Bleeding was more frequent in the ESRD with dialysis group than in the non-ESRD group. ESRD with dialysis conferred a significant risk of post-ER bleeding (odds ratio, 6.1; 95\% confidence interval, 2.7-13.6; $p<0.0001$ ). All post-ER bleeding events were controlled using endoscopic hemostasis except in 1 non-ESRD case that needed surgery.

Conclusions: ESRD with dialysis confers a bleeding risk after ER. However, all bleeding events could be managed endoscopically without sequelae. Concern about bleeding should not stop endoscopists from performing ER in ESRD patients on dialysis. Clin Endosc 2020;53:452-457

Key Words: Bleeding; Dialysis; End-stage renal disease; Endoscopic resection

Received: June 3, 2019 Revised: August 12, 2019

Accepted: September 1, 2019

Correspondence: Chan Gyoo Kim

Center for Gastric Cancer, National Cancer Center, 323 Ilsan-ro, Ilsandong-gu, Goyang 10408, Korea

Tel: +82-31-920-1620, Fax: +82-32-920-5583, E-mail: glse@ncc.re.kr ORCID: https://orcid.org/0000-0002-1651-2559

\section{Young Ju Suh}

Department of Biomedical Sciences, College of Medicine, Inha University, 27 Inhang-ro, Jung-gu, Incheon 22332, Korea

Tel: +82-32-890-2833, Fax: +82-32-890-5564, E-mail: ysuh@inha.ac.kr ORCID: https://orcid.org/0000-0002-6816-8067

(c) This is an Open Access article distributed under the terms of the Creative Commons Attribution Non-Commercial License (http://creativecommons.org/ licenses/by-nc/3.0) which permits unrestricted non-commercial use, distribution, and reproduction in any medium, provided the original work is properly cited.

\section{INTRODUCTION}

Owing to population aging, the incidence of comorbidities such as hypertension, diabetes, and end-stage renal failure is increasing. The prevalence of chronic kidney disease (CKD) is also increasing worldwide with advancing age. Patients with end-stage renal disease (ESRD), especially those who are undergoing dialysis treatment, tend to have platelet dysfunction, which increases the risk of bleeding.

The risk of mortality associated with surgery for gastric 
cancer is higher in patients with ESRD than in those without. ${ }^{1,2}$ Endoscopic resection (ER) is accepted as the current standard therapy for early gastric cancer (EGC) with minimal risk of lymph node metastasis. ${ }^{3,4}$ Although ER is a less invasive procedure than surgical treatment, the possibility of postoperative complications, such as postoperative bleeding, perforation, and infection, should be considered.

Recently, it has been suggested that more frequent bleeding after ER occurs in stage 4/5 CKD patients or dialysis patients; however, no definite conclusion could be drawn because of the exceedingly small number of cases examined. ${ }^{5,6}$ Therefore, in this study, we aimed to evaluate the association and clinical significance of bleeding after ER in ESRD patients on dialysis.

\section{MATERIALS AND METHODS}

\section{Study design and participants}

This retrospective, descriptive, 10-center study was conducted after obtaining approval from the institutional review board (CHAMC 2019-03-042). All patients provided informed consent before undergoing the procedures. All patients between the ages of 20 and 80 years who were diagnosed with EGC and scheduled for ER were enrolled in the study. A standard intravenous dose of a proton pump inhibitor was administered before ER and continued after ER. All endoscopic submucosal dissection (ESD) procedures were performed by expert endoscopists who were proficient in ER.

\section{Definitions}

Macroscopic types were classified according to the Japanese Classification of Gastric Carcinoma: type I (protruded), type IIa (superficial elevated), type IIb (flat), type IIc (superficial depressed), and type III (excavated). Types I and IIa were classified as the elevated type, types IIb and IIc as the flat type, and type III as the depressed type.?

En bloc resection was defined as resection of the neoplastic lesion in 1 piece without fragmentation. Complete resection was defined as en bloc resection with histologic confirmation of tumor-free margins (i.e., absence of tumor cells in both the horizontal and vertical margins of the specimen).

Antiplatelet agents include aspirin, thienopyridines (e.g., clopidogrel, prasugrel, ticlopidine, and ticagrelor), and other platelet aggregation inhibitors (e.g., triflusal, cilostazol, limaprost, and sarpogrelate). The patients who were taking antiplatelets stopped receiving medication 5 to 7 days before gastric ER. Restarting antiplatelet therapy was at the discretion of the attending physicians, with the consultation of a cardiologist or neurologist if necessary. However, it was difficult to recruit information on medication as it is a retrospective study.
Post-ER bleeding was defined as a bleeding sign with endoscopic confirmation. Late delayed bleeding was defined as bleeding occurring $>48 \mathrm{~h}$ after the procedure. A diagnosis of perforation was made according to a direct endoscopic observation of mesenteric fat or the presence of free air in an abdominal X-ray or computed tomography scan.

\section{Endoscopic submucosal dissection/endoscopic mu- cosal resection procedure}

Endoscopic mucosal resection (EMR) and ESD were performed using the standard methods. ${ }^{8,9}$ For EMR, the raised lesion was removed using a snare after submucosal injection. For ESD, after lesion marking, saline or hyaluronic acid with or without epinephrine $(1: 10,000)$ was injected, and a circumferential incision and submucosal dissection was performed using endoscopic instruments such as an insulation-tipped diathermic knife (KD-610L; Olympus, Tokyo, Japan). ${ }^{10}$

$\mathrm{X}$-rays were taken in all patients immediately after the procedure. When perforation was suspected clinically or radiographically, further computed tomography scans were performed. When bleeding was suspected, emergency esophagogastroduodenoscopy was performed according to the physician's judgment.

\section{Outcomes}

Bleeding was analyzed in two matched groups of patients who underwent ER for gastric neoplasia. We evaluated the method and outcomes of hemostasis.

\section{Statistical analyses}

Propensity scores to estimate the probability that patients would be selected for dialysis after ESRD were developed using logistic regression to adjust for between-group differences in baseline characteristics. Matching was performed using a 1:10 optimal matching algorithm without propensity score replacement (R-MatchIt package). Patients in the ESRD group $(n=47)$ were propensity score-matched 1:10 with patients in the non-ESRD group $(n=470)$ who underwent ER for gastric neoplastic lesions. Standardized differences were estimated for all baseline covariates before and after matching to assess pre-match imbalance and post-match balance. Standardized differences of $<10.0 \%$ for a given covariate were defined as indicative of a relatively small imbalance. For matched data, clustered comparisons were performed using generalized estimating equation analysis for post-procedural bleeding.

Statistical analysis was performed using the SAS 9.3 software package (SAS Institute Inc., Cary, NC, USA) and R software version 3.5.2 (R Foundation for Statistical Computing, Vienna, Austria; www.r-project.org). Two-sided $p$-values of $<0.05$ were considered statistically significant. 


\section{RESULTS}

\section{Baseline characteristics}

This study included a total of 7,571 patients who were diagnosed with gastric adenocarcinoma or dysplasia and subsequently underwent ER. After propensity score matching, we included 47 ESRD patients on dialysis and 470 control (non-ESRD) patients. Among the 47 ESRD patients, 39 were undergoing hemodialysis and 8 were undergoing peritoneal dialysis.

The baseline characteristics of the matched patients are summarized in Table 1. A total of 517 patients who underwent ESD or EMR were included in the study. There were no significant differences in median age (69.4 years vs. 68.9 years), proportion of men (68.1\% vs. $67.9 \%)$, and comorbidities such as hypertension, diabetes, heart disease, liver cirrhosis, and anticoagulation use between the ESRD and non-ESRD groups. With respect to the tumor characteristics of the 2 groups, there were no differences in tumor type (proportion of adenocarcinoma), lesion location, macroscopic type, tumor size, tumor depth, specimen size, procedure time, and ER method.

\section{Therapeutic outcomes and factors associated with bleeding in endoscopic resection}

Of 47 ESRD patients, 27 underwent ESD for the following reasons: EGC with absolute indications in 21 (77.8\%) patients, EGC with expanded indications in 5 (18.5\%) patients, and EGC with beyond the expanded indications in $1(3.7 \%)$ patient. The en bloc resection rate was similar between the ESRD group and the non-ESRD group ( $89.4 \%$ vs. $95.3 \%$, $p=0.14$ ).

Table 1. Clinical Characteristics of the End-Stage Renal Disease and Non-End-Stage Renal Disease Groups after Propensity Score Matching

\begin{tabular}{|c|c|c|c|c|c|c|c|}
\hline \multirow{3}{*}{ Age (yr), mean (SD) } & & \multirow{2}{*}{\multicolumn{2}{|c|}{$\begin{array}{c}\text { ESRD } \\
n=47\end{array}$}} & \multirow{2}{*}{\multicolumn{2}{|c|}{$\begin{array}{c}\text { Non-ESRD } \\
n=470\end{array}$}} & \multirow{3}{*}{$\frac{p \text {-value }}{0.71^{\mathrm{a})}}$} & \multirow{3}{*}{$\begin{array}{c}\text { Std. mean difference } \\
0.5128\end{array}$} \\
\hline & & & & & & & \\
\hline & & 69.38 & $(9.00)$ & 68.87 & $(8.88)$ & & \\
\hline \multicolumn{2}{|l|}{ Male sex, $n(\%)$} & 32 & (68.09) & 319 & $(67.87)$ & 0.98 & 0.0021 \\
\hline \multicolumn{2}{|l|}{ Hypertension, $n(\%)$} & 44 & (93.62) & 439 & $(93.40)$ & 0.96 & 0.0021 \\
\hline \multicolumn{2}{|l|}{ Diabetes, $n(\%)$} & 29 & $(61.70)$ & 283 & $(60.21)$ & 0.84 & 0.0149 \\
\hline \multicolumn{2}{|l|}{ Heart disease, $n(\%)$} & 11 & $(23.40)$ & 94 & $(20.00)$ & 0.58 & 0.034 \\
\hline \multicolumn{2}{|l|}{ Liver cirrhosis, $n(\%)$} & 2 & $(4.26)$ & 12 & $(2.55)$ & 0.49 & 0.017 \\
\hline \multicolumn{2}{|l|}{ Anticoagulation (\%) } & 23 & $(48.94)$ & 219 & $(46.60)$ & 0.76 & 0.0234 \\
\hline \multirow[t]{3}{*}{ Tumor type (\%) } & Adenocarcinoma & 27 & $(57.45)$ & 287 & $(61.06)$ & 0.74 & \\
\hline & Adenoma & 18 & $(38.30)$ & 171 & $(36.38)$ & & 0.0191 \\
\hline & Other ${ }^{c}$ & 2 & $(4.26)$ & 12 & $(2.55)$ & & 0.017 \\
\hline \multirow[t]{2}{*}{ Tumor location (\%) } & Antrum & 24 & $(51.06)$ & 246 & $(52.34)$ & 0.86 & \\
\hline & Body & 23 & $(48.94)$ & 224 & $(47.66)$ & & 0.0128 \\
\hline \multirow[t]{3}{*}{ Macroscopic type (\%) } & Elevated & 25 & $(53.19)$ & 258 & $(55.11)$ & 0.93 & \\
\hline & Flat & 9 & $(19.15)$ & 80 & $(17.02)$ & & 0.0213 \\
\hline & Depressed & 13 & $(27.66)$ & 131 & $(27.87)$ & & -0.0021 \\
\hline Tumor size & Mean (SD) & 20.40 & $(10.67)$ & 19.46 & (11.99) & 0.35 & 0.9383 \\
\hline \multirow[t]{2}{*}{ Tumor depth (\%) } & Mucosa & 45 & (95.74) & 453 & $(96.38)$ & 0.82 & \\
\hline & Submucosa & 2 & $(4.26)$ & 17 & $(3.62)$ & & 0.0064 \\
\hline Specimen size & Mean (SD) & 33 & $(10.05)$ & 33.76 & $(12.06)$ & 0.68 & -0.7611 \\
\hline Procedure time (min) & Mean (SD) & 58.19 & $(51.62)$ & 46.76 & $(49.30)$ & $0.14^{\mathrm{b})}$ & 11.4311 \\
\hline \multirow[t]{3}{*}{ Lesion size (mm) } & & & & & & 0.9745 & \\
\hline & $>20$ & 12 & $(25.53)$ & 119 & $(25.32)$ & & 0.0021 \\
\hline & $\leq 20$ & 35 & $(74.47)$ & 351 & $(74.68)$ & & \\
\hline \multirow{2}{*}{$\begin{array}{l}\text { Endoscopic resection } \\
\text { method }\end{array}$} & ESD & 45 & (95.74) & 455 & (96.81) & 0.70 & \\
\hline & EMR & 2 & $(4.26)$ & 15 & (3.19) & & 0.0106 \\
\hline
\end{tabular}

EMR, endoscopic mucosal resection; ESD, endoscopic submucosal dissection; ESRD, end-stage renal disease; SD, standard deviation. $P$-value in the chi-squared test, ${ }^{\text {a }} t$-test. ${ }^{\text {b) }}$ Mann-Whitney $U$-test. ${ }^{c)}$ Atypical cells or glands, Inflammation (gastritis, ulcer). 
The complete resection rate (87.0\%) of the ESRD group was lower than that of the non-ESRD group $(94.7 \%, p=0.04)$ (data not shown).

Post-ER bleeding was more frequent in the ESRD group than in the non-ESRD group ( $25.5 \%$ vs. $5.3 \%$ ). As shown in Table 2, ESRD with dialysis conferred a significant risk of post-ER bleeding (odds ratio, 6.1; 95\% confidence interval, $2.7-13.6 ; p<0.0001)$. This result suggests that dialysis is associated with the risk of bleeding after ER. All bleeding events in the ESRD group were successfully managed endoscopically; however, 1 patient in the non-ESRD group needed surgery. Table 3 summarizes the clinical details of 3 patients with recurrent post-ESD bleeding in the dialysis group. One of these 3 patients, who underwent dialysis on the day of ESD, experienced 3 bleeding events. The pathologic diagnosis of the lesion of this patient was poorly differentiated adenocarcinoma with submucosal invasion. All of these 3 patients were using an anticoagulant and were on hemodialysis. One patient (patient 1) had severe fibrosis. All of these cases were successfully treated endoscopically. There was no perforation in the ESRD group; however, perforation was observed in 7 patients of the non-ESRD group ( $0 \%$ vs. $1.5 \%, p=0.40$ ).

\section{DISCUSSION}

In this multicenter study, we found that ESRD with hemodialysis confers post-ER bleeding risk after controlling for endoscopic procedures.
In general surgery, patients with chronic renal failure have a higher mortality rate and longer hospital stay than those without chronic renal failure. ${ }^{11}$ Endoscopic treatment, such as EMR or ESD, is less invasive and is expected to have fewer adverse effects than surgery. However, it has been reported that more frequent bleeding after ER occurs in ESRD patients. Moreover, the safety of ER in ESRD patients on dialysis is uncertain. Goto et al. ${ }^{6}$ performed ESD in $10 \mathrm{CKD}$ patients on hemodialysis; delayed bleeding occurred in 10\% (1/10 patients) and delayed perforation (colon) in 10\% (1/10 patients). Numata et al. ${ }^{12}$ reported that the rate of post-ESD bleeding was higher in hemodialysis patients than in non-hemodialysis patients $(33 \%[5 / 15]$ vs. $9 \%[6 / 56])$. Matsumura et al. ${ }^{13}$ reported that post-ESD bleeding occurred in 20\% (4/20) of dialysis patients. Our study also showed a higher bleeding rate of $25.5 \%$ $(12 / 47)$ in ESRD patients, which was significantly higher than that in non-ESRD patients.

Bleeding could be frequent in ESRD patients because of the higher bleeding tendency, existence of cardiovascular complications, immunocompromised state, and vulnerability of tissues. Bleeding could be a serious complication after ER which can prolong hospitalization and decrease patient satisfaction. In patients with chronic renal failure, platelet dysfunction contributes to the bleeding tendency. ${ }^{14}$ Abnormal platelet-endothelium interaction also causes bleeding. Anticoagulant use during hemodialysis may contribute to bleeding risk. Further, accumulation of medications in blood in ESRD patients, owing to poor clearance, affects bleeding risk. In addition, heparin use during dialysis also increases the risk of

Table 2. Association between End-Stage Renal Disease with Dialysis and Bleeding after Endoscopic Resection after Propensity Score Matching

\begin{tabular}{|c|c|c|c|c|c|}
\hline & \multicolumn{2}{|c|}{$n(\%)$} & \multicolumn{3}{|c|}{ GEE model } \\
\hline & Post-ER bleeding & Non-bleeding & OR & 95\% CI & $p$-value \\
\hline ESRD with dialysis & $12(25.5)^{\mathrm{a})}$ & $35(74.5)^{\mathrm{b})}$ & 6.1 & $2.74-13.60$ & $<0.0001$ \\
\hline Non-ESRD & $25(5.3)$ & $445(94.7)$ & 1 & reference & \\
\hline
\end{tabular}

CI, confidence interval; ER, endoscopic resection; ESRD, end-stage renal disease; GEE, generalized estimating equation; OR, odds ratio.

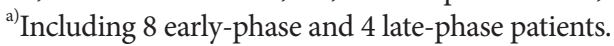

${ }^{b}$ Including 13 early-phase and 12 late-phase patients.

Table 3. Clinical and Tumor Characteristics of 3 Patients with Recurrent Post-Endoscopic Submucosal Dissection Bleeding in the Dialysis Group

\begin{tabular}{lccccccccc}
\hline $\begin{array}{l}\text { Patient } \\
\text { no. }\end{array}$ & $\begin{array}{c}\text { Age } \\
(\mathbf{y r})\end{array}$ & Sex & $\begin{array}{c}\text { Use of } \\
\text { anticoagulants }\end{array}$ & $\begin{array}{c}\text { Tumor } \\
\text { location }\end{array}$ & $\begin{array}{c}\text { Tumor size } \\
(\mathbf{m m})\end{array}$ & $\begin{array}{c}\text { Pathologic } \\
\text { result }\end{array}$ & $\begin{array}{c}\text { Dialysis } \\
\text { type }\end{array}$ & $\begin{array}{c}\text { Number of } \\
\text { bleeding }\end{array}$ & $\begin{array}{c}\text { Day of bleeding } \\
\text { after ESD }\end{array}$ \\
\hline 1 & 64 & $\mathrm{M}$ & + & Upper & 15 & $\begin{array}{c}\text { Well } \\
\text { differentiated }\end{array}$ & HD & 2 & Days 1,9 \\
2 & 69 & $\mathrm{~F}$ & + & Mid & 12 & $\begin{array}{c}\text { Well } \\
\text { differentiated }\end{array}$ & HD & 2 & Days 1, 10 \\
3 & 71 & F & + & Angle & 20 & $\begin{array}{c}\text { Poorly } \\
\text { differentiated }\end{array}$ & HD & 3 & Days 1, 2, 10 \\
\hline
\end{tabular}

ESD, endoscopic submucosal dissection; HD, hemodialysis. 
hemorrhage. ${ }^{14}$ Hemodialysis itself can contribute to bleeding, as the artificial surfaces of the dialyzer and blood tubing can cause platelet activation-inducing degranulation and loss of glycoprotein receptors. ${ }^{15}$ However, in our study, the number of patients undergoing peritoneal dialysis was too small, and it is meaningless to evaluate these patients separately from those undergoing hemodialysis.

Although bleeding is frequent in ESRD patients on dialysis, all bleeding in ESRD patients in this study was successfully managed using endoscopic procedures such as hemoclipping, epinephrine injection, electrocoagulation, and argon plasma coagulation. Further, no ESRD patient required surgery because of bleeding. Therefore, there is no reason to be reluctant to perform ER because of concern about bleeding, because the risk of mortality associated with surgery for gastric cancer is higher. ${ }^{1,2}$

In our study, there was no difference in en bloc resection rates $(89.4 \%$ vs. $95.3 \%, p=0.14)$, but complete resection rate was lower in ESRD with the dialysis group (87.0\% vs. 94.7\%, $p=0.04)$. Because we matched lesion size and depth between two groups, the proportion of beyond expanded criteria of cancer patients was not different between ESRD with dialysis $(3.7 \%, 1 / 27)$ and control group $(4.1 \%, 12 / 287)$. The reason why the complete resection rate is low in the ESRD group is unclear. A recent study comparing the outcome of ESD between patients with CKD and a control group reported no significant differences between the groups in terms of en bloc and curative resection rates. ${ }^{5}$ Whether frequent bleeding or other hidden factors in ESRD with dialysis affect complete resection may require further study.

The strength of our study is that we compared many dialysis patients with non-dialysis patients by using a propensity score-matched case-control analysis to minimize bias. To our knowledge, this study enrolled the largest number of dialysis patients undergoing ER. However, this study also has some limitations. First, this study is limited by its retrospective nature. We were unable to obtain detailed and accurate descriptions of endoscopic procedures and adverse events data. ER procedures were performed by several endoscopists; however, we could not take operator differences into account. Second, the hemodialysis protocol followed before and after ER differed in each hospital, but we could not obtain the details because of the retrospective study design. Bleeding frequency or severity may vary depending on the time of dialysis and the medication used during dialysis; however, it was impossible to analyze these factors. Third, considering the low survival rate and higher bleeding rate of ESRD patients undergoing dialysis, further studies are needed to determine if ER is necessary for adenomatous lesions.

In conclusion, ESRD with dialysis was found to confer a risk of bleeding after ER. However, all bleeding events could be managed endoscopically without sequelae. Endoscopists should not hesitate to perform ER in ESRD patients on dialysis because of concern about bleeding.

\section{Conflicts of Interest}

The authors have no financial conflicts of interest.

\section{Acknowledgements}

This study was supported by a grant from the Korean Gastrointestinal Endoscopy Research Foundation (2018 Investigation Grant) for the ESD study group. We thank members of the ESD registry (Jong Jae Park, Korea University College of Medicine Guro Hospital; Jae Myung Park, The Catholic University of College of Medicine; Moon Kyung Joo, Korea University College of Medicine Guro Hospital; Suck Chei Choi, Wonkwang University School of Medicine; Jae Kyu Sung, Chungnam National University; Jun-Hyung Cho, Soonchunhyang University Hospital; Kwang-Bum Cho, Keimyung University Dongsan Medical Center; Jae-Young Jang, Kyung Hee University) for their contributions of data for the trial.

\section{Author Contributions}

Conceptualization: Chan Gyoo Kim, Young Ju Suh

Data curation: Gwang Ho Baik, Sun Moon Kim

Formal analysis: Young Dae Kim, Chul-Hyun Lim

Investigation: Jung Won Jeon, Su Jin Hong

Methodology: Joon Sung Kim, Jun-Won Chung

Project administration: YJS

Validation: Younkyung Oh

Writing-original draft: In Kyung Yoo

Writing-review\&editing: Byoung Wook Bang

\section{REFERENCES}

1. Martínez Mier G, Alvarez-Tostado Fernández JF, Romero Hernández T, Martínez Mier EA, Blanco Benavides R. [Morbidity and mortality in surgery for gastric cancer]. Rev Gastroenterol Mex 1999;64:78-84.

2. Newman LA, Mittman N, Hunt Z, Alfonso AE. Survival among chronic renal failure patients requiring major abdominal surgery. J Am Coll Surg 1999;188:310-314.

3. Oda I, Gotoda T, Hamanaka H, et al. Endoscopic submucosal dissection for early gastric cancer: technical feasibility, operation time and complications from a large consecutive series. Dig Endosc 2005;17:54-58.

4. Ono H, Kondo H, Gotoda T, et al. Endoscopic mucosal resection for treatment of early gastric cancer. Gut 2001;48:225-229.

5. Choi YK, Ahn JY, Na HK, et al. Outcomes of endoscopic submucosal dissection for gastric epithelial neoplasm in chronic kidney disease patients: propensity score-matched case-control analysis. Gastric Cancer 2019;22:164-171.

6. Goto O, Fujishiro M, Kodashima S, et al. Feasibility of endoscopic submucosal dissection for patients with chronic renal failure on hemodialysis. Dig Endosc 2010;22:45-48.

7. Japanese Gastric Cancer Association. Japanese classification of gastric carcinoma - 2nd English edition. Gastric Cancer 1998;1:10-24.

8. Fujishiro M, Yahagi N, Kakushima N, et al. Outcomes of endoscopic submucosal dissection for colorectal epithelial neoplasms in 200 consecutive cases. Clin Gastroenterol Hepatol 2007;5:678-683; quiz 645.

9. Saito Y, Uraoka T, Matsuda T, et al. Endoscopic treatment of large superficial colorectal tumors: a case series of 200 endoscopic submucosal dissections (with video). Gastrointest Endosc 2007;66:966-973.

10. Oyama T, Tomori A, Hotta K, et al. Endoscopic submucosal dissection 
of early esophageal cancer. Clin Gastroenterol Hepatol 2005;3(7 Suppl 1):S67-S70.

11. Cherng YG, Liao CC, Chen TH, Xiao D, Wu CH, Chen TL. Are non-cardiac surgeries safe for dialysis patients? - a population-based retrospective cohort study. PLoS One 2013;8:e58942.

12. Numata N, Oka S, Tanaka S, et al. Clinical outcomes of endoscopic submucosal dissection for early gastric cancer in patients with chronic kidney disease. J Gastroenterol Hepatol 2013;28:1632-1637.

13. Matsumura T, Arai M, Maruoka D, et al. Risk factors for early and de- layed post-operative bleeding after endoscopic submucosal dissection of gastric neoplasms, including patients with continued use of antithrombotic agents. BMC Gastroenterol 2014;14:172.

14. Kaw D, Malhotra D. Platelet dysfunction and end-stage renal disease. Semin Dial 2006;19:317-322.

15. Akizawa T, Koshikawa S, Ota K, Kazama M, Mimura N, Hirasawa Y. Nafamostat mesilate: a regional anticoagulant for hemodialysis in patients at high risk for bleeding. Nephron 1993;64:376-381. 